\title{
Acadêmicos de enfermagem do interior do Amazonas em missões ribeirinhas durante a pandemia de covid-19
}

\author{
Nursing academics from the interior Amazon in riverside missions during the covid-19 \\ pandemic
}
Académicos de enfermería de la Amazonía interior en misiones ribereñas durante la pandemia del covid-19

Jéssica Karoline Alves Portugal ${ }^{1 *}$, Marcelo Henrique da Silva Reis ${ }^{1}$, Greyce Kelly Paes de Souza ${ }^{1}$, Paula Christine Feitosa de Castro ${ }^{1}$, Miguel Afonso da Costa Pontes ${ }^{1}$, Raynah Letícia Feitosa Torres $^{1}$, Laura Antônia Torres Reis ${ }^{1}$, Sibele Naiara Ferreira Germano ${ }^{1}$, Nathalie Marinho Freire ${ }^{1}$, Wendel da Silva Barros ${ }^{1}$.

\section{RESUMO}

Objetivo: Descrever as missões ribeirinhas realizadas por acadêmicos de enfermagem em parceria com o setor de Saúde Ribeirinha de um município localizado no interior do Amazonas durante o enfrentamento da pandemia de Covid-19, entre os anos de 2020 e 2021. Relato de experiência: Trata-se de um relato de experiência do tipo descritivo, obtido durante acompanhamento de acadêmicos do $8^{\circ}, 9^{\circ}$ e $10^{\circ}$ semestre da graduação em enfermagem, que estiveram presentes no enfrentamento da pandemia do novo coronavírus junto a equipes de saúde ribeirinha no interior do Amazonas, entre junho de 2020 a julho de 2021. Previamente a execução dessas atividades, a gestão local montou estratégias de planejamento dos passos a serem executados na zona rural. Considerações finais: Através da experiência com a população ribeirinha, foi possível conhecer seus costumes, culturas, estilo de vida, além do difícil acesso até as comunidades, principalmente durante a passagem de um turbulento período pandêmico, complementado pela época de grandes cheias, onde as residências ficam mais difíceis de acessar. Também foi possível estabelecer a aproximação dos futuros profissionais de saúde junto às comunidades, estando a frente no período de testagem até a imunização dos ribeirinhos.

Palavras chave: Atenção primária à saúde, Covid-19, População rural.

\section{ABSTRACT}

Objective: To describe riverside missions carried out by nursing students in partnership with the Riverside Health sector of a municipality located in the interior of Amazonas during the fight against the Covid-19 pandemic, between 2020 and 2021. Experience report: Treats This is a descriptive experience report, obtained during the follow-up of students from the 8th, 9th and 10th semester of undergraduate nursing, who were present in the fight against the new coronavirus pandemic with riverside health teams in the interior of Amazonas, between June 2020 to July 2021. Prior to carrying out these activities, the local management set up strategies for planning the steps to be taken in the rural area. Final considerations: Through the experience with the riverside population, it was possible to know their customs, cultures, lifestyle, in addition to the difficult access to the communities, especially during the passage of a turbulent pandemic period, complemented by the time of great floods, where the homes are more difficult to access. It was also possible to establish the approximation of future health professionals with the communities, being ahead in the testing period until the immunization of riverside dwellers.

Keywords: Primary health care, Covid-19, Rural population.

${ }^{1}$ Universidade Federal do Amazonas (UFAM), Coari - AM. *E-mail: jessika.portugal@gmail.com 


\section{RESUMEN}

Objetivo: Describir las misiones ribereñas realizadas por estudiantes de enfermería en alianza con el sector Riverside Health de un municipio ubicado en el interior de Amazonas durante la lucha contra la pandemia Covid-19, entre 2020 y 2021. Informe de experiencia: Trata Este es un descriptivo relato de experiencia, obtenido durante el seguimiento de los estudiantes del octavo, noveno y décimo semestre de licenciatura en enfermería, quienes estuvieron presentes en la lucha contra la nueva pandemia de coronavirus con equipos de salud de ribera en el interior de Amazonas, entre junio de 2020 y julio de 2021 Previo a la realización de estas actividades, la dirección local estableció estrategias para planificar los pasos a seguir en el área rural. Consideraciones finales: A través de la experiencia con la población ribereña, fue posible conocer sus costumbres, culturas, estilo de vida, además del difícil acceso a las comunidades, especialmente durante el transcurso de un período turbulento pandémico, complementado con la época de grandes inundaciones, donde las viviendas son de más difícil acceso. También fue posible establecer la aproximación de los futuros profesionales de la salud con las comunidades, adelantándose en el período de prueba hasta la inmunización de los ribereños.

Palabras clave: Atención primaria de salud, Covid-19, Población rural.

\section{INTRODUÇÃO}

Notícias preocupantes e até então discretas vindas da Ásia no final de 2019 e início de 2020 chegaram até a América do Sul. Havia sido descoberto um vírus extremamente contagioso, que aos poucos foi mostrando sua gravidade e letalidade, colocando as principais lideranças políticas do mundo em alerta. A veiculação de reportagens, notícias e informes da Organização Mundial de Saúde (OMS) estava cada vez mais em evidência, a pandemia de Covid-19 passou a ser o assunto mais falado em todos os veículos de comunicação global (SENHORAS EM, 2020).

Em pouquíssimo tempo o novo coronavírus conseguiu chegar em todos os continentes, neste contexto, alguns países de imediato utilizaram medidas de restrições a fim de frear a propagação do vírus, outros duvidaram da gravidade informada pela OMS, acarretando em um grande número de infectados e uma quantidade significativa de óbitos. O surgimento da pandemia trouxe consigo impactos incalculáveis na atividade econômica das principais potências mundiais, além de impactar diretamente na saúde mental e vida das pessoas ao redor do mundo (SENHORAS EM, 2020; WORLD HEALTH ORGANIZATION (WHO), 2020).

No Brasil, o Amazonas foi um dos primeiros estados a anunciar o risco de colapso nos serviços de saúde, devido a superlotação nos hospitais, ausência de leitos e materiais. Desta forma, o Ministério da Saúde declarou situação de emergência para transmissão do coronavírus (SENHORAS EM, 2020). Com o rápido avanço do vírus por todo estado, não demorou para que regiões ribeirinhas do Amazonas começassem a registrar os primeiros casos da doença (CASTRO FF, et al., 2020).

A região amazônica é rica em biodiversidade e abriga a maior floresta tropical do planeta. O Amazonas é o maior estado brasileiro, com aproximadamente $1.559 .148 \mathrm{~km} 2$ e baixa densidade demográfica - 2,2 habitantes por km2. A densa vegetação e extensas bacias hidrográficas, explicam as características da região (INSTITUTO BRASILEIRO DE GEOGRAFIA E ESTATÍSTICA (IBGE), 2010; IBGE, 2021).

Essas regiões possuem sérias limitações econômicas e de acesso aos recursos de saúde, e apesar do grande avanço da medicina moderna, ainda existem muitos obstáculos para a assistência à saúde. A vida na área rural da região amazônica apresenta diversos desafios não encontrados em grandes metrópoles, como a inexistência de saneamento básico, condições de saúde inadequadas, atividades rotineiras árduas devido ao trabalho no campo e a falta de informações sobre o autocuidado, propiciando o surgimento de inúmeros problemas de saúde (FRAXE TJP, et al., 2007; GAMA ASM, 2016; SOUSA IS, 2009).

Para a utilização dos serviços de saúde, os ribeirinhos precisam deslocar-se de sua comunidade até a sede do município, esperar a passagem da Unidade Básica de Saúde Fluvial, Barcos Missionários ou contar com o apoio do Agente Comunitário de Saúde (ACS). Estas limitações impõem dificuldades enormes, que fazem com que as populações ribeirinhas busquem alternativas para o tratamento de eventuais enfermidades, 
como a utilização da medicina caseira por meio do uso de plantas medicinais cultivadas em seus quintais e também pela utilização de medicamentos alopáticos facilmente adquiridos sem prescrição nas farmácias da zona urbana (FRANCO EC, et al., 2015; GAMA ASM, et al., 2018; REIS MHS, et al., 2021).

Diante do crescente avanço da pandemia, as comunidades ribeirinhas vivenciaram completo estado de calamidade na saúde pública. Desta forma, surge um novo desafio: conter o avanço da doença, rastrear e tratar indivíduos contaminados para evitar evolução do quadro de gravidade e consequente hospitalização (CASTRO FF, et al., 2020).

Com a realidade imposta forçadamente pela pandemia, os principais serviços não essenciais para a sobrevivência humana foram suspensos, muitas classes de trabalhadores foram orientadas a ficarem em suas casas, evitando que o vírus se alastrasse, principalmente em grandes centros. No entanto, enquanto o mundo se recolheu, diversos profissionais de saúde foram convocados para uma guerra invisível, atuando em hospitais e demais serviços de saúde, arriscando suas vidas e enfrentando situações adversas que prejudicaram sua saúde física e suas mentes, além disto, muitos ainda tiveram que lidar com a perda de entes queridos e colegas de trabalho (THE LANCET, 2020).

O Ministério da Educação (MEC), mediante ao grande número de profissionais de saúde afastados de suas funções, publicou a Portaria ํㅡ 356, em 20 de março de 2020, que dá permissão para alunos da área de saúde atuarem no combate a pandemia de Covid-19 (MINISTÉRIO DA EDUCAÇÃO, 2020). Diante do exposto, o objetivo deste estudo foi descrever as missões ribeirinhas realizadas por acadêmicos de enfermagem em parceria com o setor de Saúde Ribeirinha de um município localizado no interior do Amazonas durante o enfrentamento da pandemia de Covid-19, entre os anos de 2020 e 2021.

\section{RELATO DE EXPERIÊNCIA}

Trata-se de um relato de experiência do tipo descritivo, obtido durante acompanhamento de acadêmicos do $8^{\circ}, 9^{\circ}$ e $10^{\circ}$ semestre da graduação em enfermagem, que estiveram presentes no enfrentamento da pandemia do novo coronavírus junto a equipes de saúde ribeirinha no interior do Amazonas, entre junho de 2020 a julho de 2021. Previamente a execução dessas atividades, a gestão local montou estratégias de planejamento dos passos a serem executados na zona rural.

Em relação ao acompanhamento de saúde, todas as comunidades e localidades possuem como porta de entrada preferencial os seguintes locais: Unidade Básica de Saúde do Ribeirinho, que possui 02 equipes de estratégia saúde da família, a Unidade Básica de Saúde Fluvial que está credenciada na calha do médio Solimões, mas percorre todas as comunidades durante o ano. Cada comunidade ribeirinha ou agrupamento de duas ou três comunidades menores, possui um ACS morador local (REIS MHS, et al., 2021).

No ano de 2020, mesmo sem ter nenhum caso confirmado ou suspeito, a gestão municipal já preparava o corpo de saúde para prestar assistência aos pacientes que se contaminassem com o vírus, adotando medidas primordiais para o contingenciamento que foram adotadas em outros estados do país, além de fechar os estabelecimentos não essenciais, visto que era questão de tempo para o primeiro caso ser confirmado, ainda mais numa época em que o transporte fluvial foi paralisado e inúmeras embarcações com passageiros estavam chegando clandestinamente da capital, onde os casos estavam em ascensão (PORTUGAL JKA, et al., 2020).

Por se tratar de um vírus altamente contagioso, mesmo seguindo todas as medidas de segurança e cautela possíveis, no mês de abril foi diagnosticado o primeiro caso confirmado no município, trazendo pânico a toda população local. A vítima veio a óbito dias depois de chegar à cidade, vinda numa embarcação clandestina e partir daí o risco que era iminente tornou-se uma assombrosa realidade. Entre meados de abril a final de maio, ocorreu a confirmação de milhares de casos e centenas de mortes num intervalo de 45 dias, um cenário apocalíptico.

Com todas as adversidades, a saúde continuou sua árdua luta, neste período dezenas de profissionais se contaminaram e foram afastados por atestado médico. As equipes estavam desfalcadas, neste contexto, a Secretaria de Saúde passou a contar com o suporte de 20 acadêmicos de enfermagem ainda em 2020 e a 
missão a eles impostas foi a cobertura dos povos da zona ribeirinha. Além dos discentes, a equipe foi formada por médicos, enfermeiros, técnicos em enfermagem, agentes comunitários de saúde e demais classes profissionais de diferentes níveis (JÚNIOR JCFP, et al., 2020).

Todas as viagens realizadas tiveram suporte de embarcações de grande potência, para que pudessem chegar nas áreas mais distantes em tempo hábil, além de serem primordiais para o retorno ainda com a luz do dia, garantindo a segurança da equipe em áreas tão atingidas pelo crime organizado presente nos rios da Amazônia. Cada missão tinha duração de 1 dia, contando com suporte de até 12 tripulantes, entre profissionais e voluntários.

As escolas e centros comunitários serviam de pontos de apoio nas comunidades com terra firme presente, onde não existia essa possibilidade a própria embarcação era utilizada como base de atendimentos. Dentre as atividades desenvolvidas, podemos destacar: ações de educação em saúde, entrevistas com a notificação em massa de todos os ribeirinhos que tivessem interesse em realizar a testagem do Covid-19, além da busca específica das comorbidades (JÚNIOR JCFP, et al., 2020).

As testagens foram realizadas em dois cenários: coleta e entrega do diagnóstico de imediato nas viagens em que o bioquímico estava presente e coleta de sangue para entrega posterior em tempo mínimo ao agente comunitário de saúde ou liderança local. Além da ação de testagem, foram realizadas as entregas de máscaras e kits de higiene. Os ribeirinhos com resultados positivos e negativos receberam atendimento médico e de enfermagem conforme a demanda.

A vacina ainda era um sonho distante no ano de 2020, as ações realizadas até janeiro de 2021 consistiram principalmente na testagem, identificação dos casos positivos e posterior isolamento social a fim de evitar que mais indivíduos se contaminassem. Neste período foram realizados mais de 5.000 testes nas populações ribeirinhas que aceitaram tal conduta, pois ainda existiam diversos comunitários que se recusavam a realizar teste rápido, algo surreal que foi visto novamente com o advento da vacina.

As primeiras doses de imunização contra a covid-19 chegaram no Amazonas em janeiro de 2021, em pouco tempo foram distribuídas pequenas quantidades para os municípios do interior, com objetivo de atender as demandas conforme direcionamento do Ministério da Saúde. Prioritariamente foram vacinadas as pessoas acima de 75 anos, pois apresentavam maiores fragilidades e estavam nas estatísticas como a faixa etária mais atingida em relação a óbitos.

Com o passar dos meses a idade prioritária foi caindo, principalmente no Amazonas, que registrava índices elevados de superlotação de hospitais e mortes. Em abril de 2021, a vacinação estava disponível para todos os ribeirinhos de 18 anos ou mais que comprovassem residir em áreas rurais. Entre fevereiro a julho de 2021, foram vacinados em torno de 5.600 indivíduos em todas as missões ribeirinhas e mutirões realizados no entorno da cidade, esse quantitativo poderia ser facilmente dobrado, mas diversos empecilhos dificultaram o trabalho das equipes de saúde.

A influência das correntes de redes sociais, fake news, medo, crenças e posicionamento político foram os principais motivos das recusas encontradas e relatadas por profissionais de saúde que lideraram as missões.

\section{DISCUSSÃO}

Conforme rege um dos princípios do Sistema Único de Saúde (SUS), o acesso à saúde é universal, sem discriminação entre pobre, rico, branco e negro. Toda população do território nacional tem direito de desfrutar dos serviços de saúde disponíveis (BRASIL, 1990).

De modo geral, os ribeirinhos são pessoas de baixa renda e escolaridade, carência de informações, muitos estão isolados a ponto de não terem eletricidade e acesso a qualquer meio de comunicação, fato que os afasta de qualquer tipo de notícias do âmbito local, regional, nacional e mundial (GAMA ASM, et al., 2018; GUIMARÃES AF, et al., 2020). Foi identificado em várias comunidades indivíduos que na pandemia fizeram um maior uso de plantas medicinais para tratar sintomas da covid-19, além do uso de medicamentos sem a presença de nenhum sinal de enfermidade, a fim de usar na profilaxia para eventual contaminação, esse achado pode estar atrelado ao baixo nível educacional e pouco acesso à informação. 
Apesar de considerarmos uma prática inadequada, desde os primórdios da humanidade, o homem sempre procurou amenizar ou eliminar sofrimentos causados por sintomas indesejados, com características peculiares a cada época e a cada região, buscando auxílio em plantas e ervas medicinais. Com o passar dos anos, o avanço da medicina e a descoberta de substâncias químicas presentes em plantas, o homem começou a não se restringir apenas aos produtos naturais para melhoria da saúde, passando a consumir medicamentos produzidos e aperfeiçoados pelas grandes indústrias farmacêuticas, com o objetivo de lhe trazer benefícios no tratamento de doenças, alívio de sintomas e na promoção do bem-estar (FONSECA FIRM, et al., 2010).

Por mais que faça parte da cultura herdadas pelos antepassados, essas informações nos colocam em um grande alerta, pois não existem muitas evidências científicas que o uso de grande parte das plantas medicinais tenha a eficácia que os mais experientes afirmam. O uso de algo desconhecido pode levar a intoxicações e trazer complicações a saúde do indivíduo, assim como a utilização de medicamentos sem o direcionamento do profissional habilitado (GAMA ASM, 2016).

Em relação ao público alvo das viagens, destaca-se o sexo feminino, correspondendo a mais de $60 \%$ dos testados e vacinados. Esta informação é similar a outros estudos envolvendo populações ribeirinhas locais e pode ser justificado pelo fato de as mulheres possuírem algumas diferenças fisiológicas em relação aos homens, que podem influenciar numa maior busca pelos serviços de saúde disponíveis, além de serem consideradas as gestoras da saúde de sua família e terem mais disciplina em relação aos exames periódicos e consultas de rotina, desta forma, estão propensas a procurarem mais os serviços de saúde do que os homens (GAMA ASM, et al., 2018; GUIMARÃES AF, et al., 2020; REIS MHS, et al., 2020).

Tendo em vista o grande desfalque de profissionais nos serviços de saúde, a tomada de decisão do Ministério da Educação com posterior vinda dos acadêmicos para integrar as equipes de saúde foi importantíssima, visto que são indivíduos em formação e com vontade de mostrar serviço para adquirir experiências que não são apresentadas na academia. São oportunidades com potencial de moldar esses futuros enfermeiros, Ihes mostrando as dificuldades que a vida profissional impõe, principalmente em nosso país que possui um sistema de saúde gratuito e capaz de chegar onde nenhum outro chega, porém, encontrase defasado, por conta da má gestão na administração dos recursos oriundos dos impostos e a falta de investimentos (MILLER DG, et al., 2020).

Através da experiência com a população ribeirinha, foi possível conhecer seus costumes, culturas, estilo de vida, além do difícil acesso até as comunidades, principalmente durante a passagem de um turbulento período pandêmico, complementado pela época de grandes cheias, onde as residências ficam mais difíceis de acessar. Também foi possível estabelecer a aproximação dos futuros profissionais de saúde junto às comunidades, estando a frente no período de testagem até a imunização dos ribeirinhos, colocando em prática os conhecimentos adquiridos durante a graduação que está em curso, praticando a educação em saúde, elucidando dúvidas a respeito do cuidado com a saúde e compartilhando experiências junto a esses indivíduos que vivem isolados geograficamente. Essa participação foi primordial para ressaltarmos o grande valor que o SUS tem para a população ribeirinha, garantido o acesso à saúde aos povos que tem os rios como suas "estradas líquidas".

\section{REFERÊNCIAS}

1. BRASIL. 1990. Lei no 8.080, de 19 de setembro de 1990. Lei Orgânica da Saúde. Dispõe sobre as condições para a promoção, proteção e recuperação da saúde, a organização e o funcionamento dos serviços correspondentes e dá outras providências. Brasília. Disponível em: http://www.planalto.gov.br/ccivil_03/leis/l8080. Acessado em: 10 de novembro de 2021.

2. CASTRO FF, et al. Idosos ribeirinhos da Amazônia Brasileira no enfrentamento da covid-19. 2ª ed. Brasília: Editora ABEn, 2020; 142-149p.

3. FONSECA FIRM, et al. Frequência de automedicação entre acadêmicos de faculdade de medicina. Diagn Tratamento, 2010; 15(2): 53-57. 
4. FRANCO EC, et al. Promoção da saúde da população ribeirinha da região amazônica: relato de experiência. Rev CEFAC, 2015.

5. FRAXE TJP, et al. Comunidades ribeirinhas amazônicas: modos de vida e uso dos recursos naturais. Manaus: EDUA; 2007.

6. GAMA ASM. Automedicação em comunidades ribeirinhas na região do Médio Solimões, Amazonas. Tese (Doutorado em Enfermagem na Saúde do Adulto). Universidade de São Paulo (USP), São Paulo, 2016; 115 p.

7. GAMA, ASM, et al. Inquérito de saúde em comunidades ribeirinhas do Amazonas, Brasil. Cad. Saúde Pública, 2018.

8. GUIMARÃES AF, et al., Acesso a serviços de saúde por ribeirinhos de um município no interior do estado do Amazonas, Brasil. Rev Pan Amaz Saude, 2020.

9. INSTITUTO BRASILEIRO DE GEOGRAFIA E ESTATÍSTICA (IBGE). 2021. Cidades: informações completas. Disponível em: http://cidades.ibge.gov.br/xtras. Acessado em: 10 de novembro de 2021.

10. INSTITUTO BRASILEIRO DE GEOGRAFIA E ESTATÍSTICA (IBGE). 2010. Sinopse do censo demográfico de 2010. Disponível em: http://cidades.ibge.gov.br/xtras. Acessado em: 10 de novembro de 2021.

11. JÚNIOR JCFP, et al. Atuação de acadêmicos de enfermagem nas ações de enfrentamento da covid-19 em comunidades ribeirinhas do amazonas: relato de experiência. Revista eletrônica Acervo Saúde, 2020.

12. SENHORAS EM. Novo Coronavírus e seus impactos econômicos no mundo. Boletim de Conjuntura (BOCA), 2020.

13. MILLER DG, et al. The role of medical students during the COVID-19 pandemic. Ann Intern Med. 2020.

14. MINISTÉRIO DA EDUCAÇÃO. 2020. Portaria n. 356, de 11 de março de 2020. Disponível em: https://www.in.gov.br/en/web/dou. Acessado em: 10 de novembro de 2021.

15. PORTUGAL JKA, et al. Percepção do impacto emocional da equipe de enfermagem diante da pandemia de COVID19: relato de experiência. Revista eletrônica Acervo Saúde, 2020.

16. REIS MHS, et al. Características da população ribeirinha de um município do interior do Amazonas. Revista eletrônica Acervo Saúde, 2021.

17. REIS MHS, et al. O impacto do advento de uma Unidade Básica de Saúde Fluvial na assistência aos povos ribeirinhos do Amazonas. Revista eletrônica Acervo Saúde, 2020.

18. SOUSA IS. As condições de vida e saneamento nas comunidades da área de influência do gasoduto Coari-Manaus em Manacapuru - AM. Hygeia. 2009;5(9):88-98.

19. THE LANCET. COVID-19: protecting health-care workers. Editorial Lancet, 2020; 395: 922.

20. WORLD HEALTH ORGANIZATION (WHO). 2020. Doença por coronavírus 2019 (COVID-19): relatório de situação 36. Genebra. Disponível em: https://www.who.int/docs/default-source/coronaviruse/situation-reports/20200225. Acessado em: 10 de novembro de 2021. 